\title{
Flip-flop chaining architecture for power-efficient scan during test application
}

\author{
Shantanu Gupta and Tarang Vaish \\ Department of Computer Science \\ and Engineering, IIT Guwahati \\ North Guwahati, Assam - 781039 \\ gshantanu@gmail.com, tarang@iitg.ernet.in
}

\author{
Santanu Chattopadhyay \\ Department of Electronics and \\ Electrical Communication Engineering, \\ IIT Kharagpur, West Bengal - 721302 \\ santanu@ece.iitkgp.ernet.in
}

\begin{abstract}
Power dissipation in CMOS circuits during test time poses a crucial bottleneck for circuit performance and robustness. The power consumption due to switching activity while scan-in of test vectors and scan-out of responses is of particular concern. In this paper a methodology for scan chain modification and test vector adaptation is proposed to effectively reduce the scan test power consumption by controlling this switching activity. Proposed approach, unlike the many in published literature, does not incorporate reordering of scan cells; thus avoiding timing and routing overheads. ATPG software ATALANTA was used for test vector generation. The algorithm was verified for ISCAS' 89 benchmark circuits, where it showed as much as $27.3 \%$ of reduction in switching activity during scan operations.
\end{abstract}

\section{Introduction}

Power dissipation is a crucial issue because of its wide ranged effects on circuit performance and life of circuit under test (CUT). Exceeding peak power limitations of any circuit can cause irreversible damages. During the test, scan-in/scan-out of vector/response causes excessive switching activity. In CMOS circuits average test power consumption is much higher than the normal mode operation and is directly proportional to this switching activity [3]. Several techniques have been proposed to reduce the same. A rather popular technique - addition of extra design for test (DfT) logic - helps in reducing the switching activity but adds area overhead. Many works [3,5] discuss heuristics for test vector re-ordering. Works involving scan cell re-ordering (or scan-latch ordering) [2,7,9] have also been prevalent in the literature; but these face the criticism for their test timing inconsistency and decoder buffer problem. Finally, methods have been explored which involve scan chain partitioning that reduce the scan chain lengths [10] but at the same time induces hardware overheads. In a nutshell, most of the current day techniques for power aware testing are inflicted with: (1) additional DfT logic overhead, (2) test timing inconsistency due to re-ordering of scan cells, and (3) decoder buffer problems due to reordering of scan cells.

Evidently, an approach that does not involve the use of an additional DfT logic will be ridden of problem 1. And avoiding scan cell re-ordering will take care of problem 2 and 3. The proposed approach, named as Optimized Scan Cell Testing (OSCT), fits exactly in this description. And thus eliminates all of the aforementioned problems. The algorithm does not interfere with the layout decision and thus it can be optimized separately. At all times fault coverage and test time of the circuit are left unaffected. The work concentrates only upon the reduction of scan chain (flip-flop cells) switching activity as it forms a significant part of the total power consumed during scan-in. The switching activity in the combinational circuit is completely ignored. The rest of the paper is organized as follows, section 2 discusses the conventional scan cell testing method (CSCT). This sets the foundation for explanation of proposed approach named as optimized scan cell testing (OSCT) in section 3, section 4 lists the simulation results obtained for ISCAS' 89 benchmark circuits. Section 5 concludes the work, followed by important references.

\section{Conventional Scan Cell Testing : CSCT}

Conventional scan cell testing is a well documented technique. [1] deals thoroughly with the subject at hand. Transitions in the scan cells result from scan-in/scan-out of test vectors/responses. Transitions also result from a clash that can be defined as a condition where the MSB of predecessor's response differs from the LSB of successor's vector. The total number of transition count is therefore given by (1) [12]. Clashes can therefore be effectively reduced in number by test vector re-ordering. We shall use this observation in section 3.3. 


$$
\begin{aligned}
\text { Total Transitions }= & \sum_{\text {Position of Transition })+}(\text { Size of Chain }- \\
& \text { Size of Chain } \times \text { Clashes }
\end{aligned}
$$

\section{Optimized Scan Cell Testing: OSCT}

In this section we propose a new approach that effectively reduces the number of scan chain transitions during the scan cell testing. The input to the algorithm is a CUT and its corresponding test pattern set. Output is a modified scan cell architecture and a test pattern set that gives optimal reduction in test power consumption. Our approach can fundamentally be divided into three steps. The first step of the algorithm does a modification of the scan chain architecture. This modification neither involves addition of a DfT logic nor does it involve scan cell re-ordering. Second step deals with the customization of test vectors for the modified scan cell architecture. In this step, we basically harness the benefits of effectively specifying the don't care bits of test vectors. In third and final step of the algorithm we do a simple test vector re-ordering that does the job of reducing total number of clashes and consequently the overall number of transitions. The approach taken in the final step for test vector re-ordering is independent of the previous two algorithmic steps.

\subsection{Scan Architecture Modification (SAM)}

Conventional way of connecting two consecutive flipflops in a scan chain involves connecting the Q (output of predecessor) to $\mathrm{D}$ (input of successor). We make a simple modification to this approach by allowing $\bar{Q}$ (negated output of predecessor) connection to $\mathrm{D}$ (input of successor). This latter type of connection is selectively done at various positions within the scan chain. During the scan-in/scanout of vectors/responses, any two differing consecutive bits within the vector/response cause flip-flop transition at every clock tick. These transitions can be reduced in number if we set up the flip-flop interconnections optimally. Any modification of the scan cell architecture will necessitate adaption of test vectors such that after scan-in they take the original form. This is dealt with in section 3.2.2.

Our objective is to make a scan architecture for effective handling of all the given test patterns, i.e. we must consider all test vector-response pairs together and decide connections (at each flip-flop junction) that lead to best possible overall reduction in the number of transitions. The test patterns are pre-determined using a suitable ATPG (ATALANTA in our case). A significant number of bit positions in the vectors/responses remain unspecified, or are don't care in nature. Ignoring these don't care bits, that are tackled later in section 3.2.1, we can compute the cost at every index $i$ of the scan chain while having 1) $Q-D$ connection and 2) $\bar{Q}-D$ connection. The calculation given by (2) decides an optimal connection type for every scan chain index.

$$
\begin{aligned}
\text { Cost }_{10,01}^{i}= & \text { VBitTotal }_{10,01}^{i} \times i+ \\
& \text { RBitTotal }{ }_{10,01}^{i} \times(\text { Size of Chain }-i) \\
\text { Cost }_{11,00}^{i}= & \text { VBitTotal }{ }_{11,00}^{i} \times i+ \\
& \text { RBitTotal }{ }_{11,00}^{i} \times(\text { Size of Chain }-i) \\
F F_{\text {connection }}^{i}= & \text { Cost }_{11,00}^{i} \geq \text { Cost }_{10,01}^{i} \\
& \forall i \in\{1, \text { Size of Chain }-1\}
\end{aligned}
$$

In (2), $i$ is the index of the flip-flop junction in the scan chain, i.e. junction between $i^{t h}$ and $i+1^{t h}$ flip-flop. $V$ BitTotal ${ }_{10,01}^{i}$ is the variable for number of times the consecutive bits differ at position $i$ when considering all the test vectors. RBitTotal $l_{10,01}^{i}$ is the variable for number of times the consecutive bits differ at position $i$ when considering all the test responses. VBitTotal ${ }_{11,00}$ and RBitTotal ${ }_{11,00}^{i}$ are similarly defined when consecutive bits are same. For each of these variables we leave out the count for don't care bits. Size of Chain is the number of flip-flops in the scan chain. Cost $_{10,01}^{i}$ stands for the number of transitions that will take place due to consecutive bits at indexes $i$ and $i+1$ while keeping flip-flop connection as $Q-D$. Similarly, $\operatorname{Cost}_{11,00}^{i}$ is similarly defined while flip-flop connection is kept as $\bar{Q}-D$. FF Fonnection is assigned a boolean value true or false whichever favors a lower value for transition cost. True imposes a $Q-D$ connection, whereas a false imposes a $\bar{Q}-D$ connection.

\subsection{Test Vector Customization (TVC)}

Test vector customization is responsible for the following tasks:

- Taking the optimized scan cell architecture as a reference, it specifies the don't care bits in the test vectors.

- It takes the test vectors and adapts them to the optimized scan cell architecture such that after scanning in they assume desired original form.

\subsubsection{Specifying the don't care bits}

ATPGs provide partially specified vectors that can be used to our advantage by customizing them for the optimized scan cell architecture. Therefore, we can do an intelligent fill-up of the don't care bits in the test vectors such that they incur lower transitions costs as compared to a situation where the don't care bits are randomly specified. The algorithm proposed handles one test vector at a time. We shall 


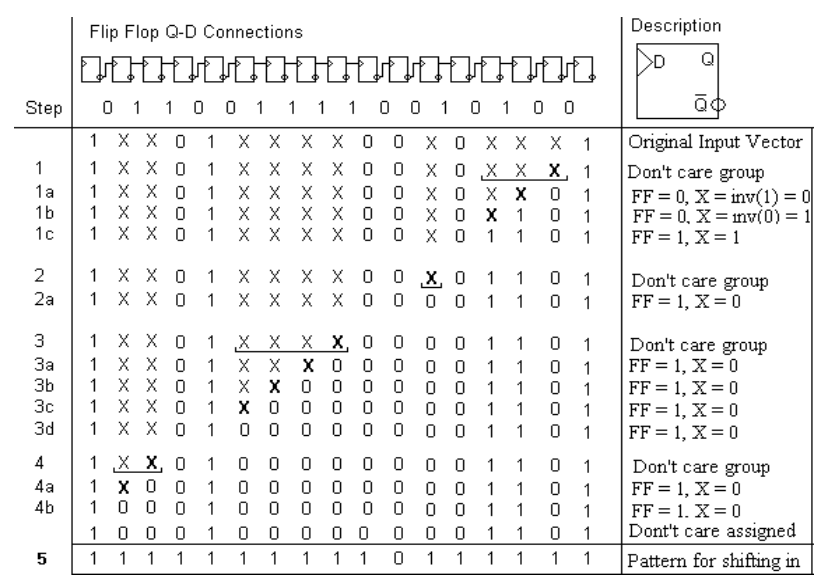

\section{Figure 1. Illustration of Test Vector Cus- tomization}

explain this algorithm with the help of a simple example, see figure 1. Alphabet $\mathrm{X}$ is used to represent a don't care bit. $Q-D$ and $\bar{Q}-D$ type of flip-flop (FF) interconnections are encoded as 1 and 0 respectively. Following steps summarize the algorithm:

1. Start from last bit (LSB) of the test vector and move toward the first bit (MSB).

2. While moving leftwards in the test vector, identify the first don't care bit, call this position $\mathbf{S}$. See step 1 in figure $1, \mathbf{X}$ is the first don't care bit.

3. Consider the right hand immediate neighbor bit of position $\mathbf{S}$, call this bit $\mathbf{R}$. In our example $\mathbf{R}=1$ for the first don't care bit.

4. Check out the connection type between the flip-flops that correspond to positions $\mathbf{S}$ and $\mathbf{R}$ of the test vector.

- If we have a $Q-D$ flip-flop connection $(F F=$ $1)$, then $\operatorname{assign} \mathbf{S}=\mathbf{R}$.

- If we have a $\bar{Q}-D$ flip-flop connection $(F F=$ $0)$, then assign $\mathbf{S}=$ inverse $(\mathbf{R})$.

Refer to steps 1a, 1b, 1c, 2a etc. in the figure 1 to get acquainted with this procedure. If the last bit in the test vector is don't care in nature, set it to a default binary value.

5. If $\mathbf{S}$ is the first bit of the test vector, stop the algorithm, otherwise go back to step 2 of this algorithm.

The test vector obtained at step $4 \mathrm{~b}$ of the figure 1 is completely devoid of don't care bits.

\subsubsection{Adaptation of test vectors}

At this stage of the algorithm, we have an optimized scan cell architecture with completely specified test vectors. But the modified scan architecture demands a change in test vectors to nullify the transitions caused by $\bar{Q}-D$ connections during scan-in. This is important as we want the test vectors to be in their original form after complete scan-in. We can handle this problem in a straightforward manner; flip a bit in the original test vector if it goes through an odd number of $\bar{Q}-D$ connections, and keep it unaltered otherwise. An example of such an adaptation of a test vector is shown in figure 1 . Step $4 \mathrm{~b}$ in the figure 1 shows a completely specified test vector. And step 5 shows an adapted test vector that is ready for a scan-in.

\subsection{Test Vector Reordering (TVR)}

In this last and final step of our algorithm, we optimally re-order the test patterns to reduce the scan chain switching transitions further. This essentially removes the clashes. Following steps summarize the algorithm for test vector reordering:

1. Attach a tag XY to every test pattern; where $X$ is LSB of the test vector, and $Y$ is MSB of the output response.

2. Four types of tags are possible: 00, 01, 10 and 11. Divide the complete set of test patterns into a maximum of 4 groups on the basis of these tags.

3 . List all the test patterns in the group 00 .

4. Pick test patterns from group 01 and 10 alternately. If any one of these two groups exhausts, list all the remaining patterns from the other group ignoring the alternate picking policy.

5. List all the test patterns in the group 11 .

The algorithm shown above is based on a simple observation. If a test pattern $A$ has a $\operatorname{tag} \mathrm{X}_{A} \mathrm{Y}_{A}$, and another test pattern $B$ has a tag $\mathrm{X}_{B} \mathrm{Y}_{B}$; then $A$ followed by $B$ would cause a clash if and only if $\mathrm{Y}_{A} \neq \mathrm{X}_{B}$. This follows directly from the definition of a clash: $\mathrm{Y}_{A}$ is MSB of the predecessor's response and $\mathrm{X}_{B}$ is LSB of successor's vector. In the algorithm we minimize the number of such occurings.

At the end of three simple steps, we have arrived at an optimized scan cell architecture and suitably customized test vectors that have been optimally ordered to reduce the number of scan chain transitions.

\section{Simulation Results and Discussions}

The complete algorithm was implemented in $\mathrm{C}$ and verified on ISCAS' 89 benchmark circuits [4]. The test patterns were generated using ATALANTA [6]. FSIM fault simulator [11] was used to obtain output responses for the customized test vectors. Table 1 summarizes the results obtained. CSCT column lists the number of transitions for complete testing when using approach from section2, SAM \& TVC (section 3.1 and section 3.2) column lists the number of transitions obtained after scan architecture modification and test vector customization. The following column (\% Imp) shows percentage improvement for this part. The 


\begin{tabular}{|c|c|c|c|c|c|c|c|c|}
\hline Circuit & \#Gates & Fault Coverage & CSCT & SAM \& TVC & \% Imp. & TVR & \% Imp. & \% Imp. [9] \\
\hline s298 & 119 & $100.00 \%$ & 2926 & 2702 & $7.65 \%$ & 2534 & $13.39 \%$ & $10 \%$ \\
\hline s510 & 211 & $100.00 \%$ & 1115 & 1057 & $5.20 \%$ & 913 & $18.12 \%$ & $5.3 \%$ \\
\hline s526 & 193 & $99.82 \%$ & 12537 & 11383 & $9.21 \%$ & 10774 & $14.06 \%$ & - \\
\hline s713 & 393 & $93.46 \%$ & 8826 & 7712 & $12.62 \%$ & 7674 & $13.05 \%$ & $17 \%$ \\
\hline s953 & 395 & $100.00 \%$ & 27905 & 24211 & $13.24 \%$ & 24095 & $13.65 \%$ & - \\
\hline s1238 & 508 & $94.91 \%$ & 22625 & 19553 & $13.58 \%$ & 19193 & $15.17 \%$ & - \\
\hline s1488 & 653 & $100.00 \%$ & 1953 & 1953 & $0.00 \%$ & 1683 & $13.82 \%$ & - \\
\hline s9234 & 5597 & $93.48 \%$ & 9775460 & 7130370 & $27.06 \%$ & 7105472 & $27.31 \%$ & $22.4 \%$ \\
\hline s15850 & 9775 & $96.68 \%$ & 64559689 & 50710629 & $21.45 \%$ & 50619849 & $21.59 \%$ & - \\
\hline
\end{tabular}

Table 1. Simulation results

second to last column TVR (section 3.3) reflects the number of transitions after application of the complete algorithm, i.e. after test vector re-ordering. The second to last column shows overall saving in scan chain power consumption for various benchmark circuits. [9] is fairly recent work that makes use of scan cell ordering approach to reduce test power and, therefore, has the problems associated with this category of approaches. A comparison with [9] hence forms an ideal ground for this work. The available values from [9] in table 1 show our method to be competetive even against a popular, but overhead ridden, scan cell re-ordering based method.

\section{Conclusions}

In this paper, we have presented a robust and efficient algorithm to reduce the scan test power consumption in CMOS circuits. Our method explores the capability of flipflops to provide both $Q$ and $\bar{Q}$ as outputs simultaneously, thus allowing us to modify the scan architecture for an efficient handling of test patterns. We also exploit the unspecified or the don't care bits in a test vector to customize it; thus giving us better performance with the optimized scan cell architecture. Apart from these novel propositions, we also do a simple test vector re-ordering. This is independent of the rest of the algorithm and therefore gives a flexibility to make future modifications and/or to employ aother test vector re-ordering scheme published in literature. Proposed method has no penalty on the fault coverage, IC test time or circuit performance. No routing issues as the scan cell remain in their original order. Finally, it is very easy to use in a classical DfT flow and has therefore a very low impact on the system design time.

\section{References}

[1] A. Crouch, Design-for-Test for Digital IC's and Embedded Core Systems, Number ISBN 0-13-08427-1 Prentice Hall, 1999.
[2] V. Dabholkar and S. Charkravarty, Techniques for minimizing power in scan and combinational circuits during test application, IEEE Trans, on Computer Aided Design, 17(12):13251333,1998

[3] Devadas and S. Malik, A survey of optimization techniques targeting low power VLSI circuits, In Proc. Of Design Automation Conferences, pages 242-247, 2002.

[4] D.Bryan F.Brglez and K.Kozminski, Combinational profiles of sequential Benchmark circuits, IEEE ISCAS, 3:1929-1934, May 1989.

[5] S. Gerstendorfer and H.J.Wunderlich,Minimized power consumption for scan-based Bist, In Proc. IEEE International Test Conference Pages 77-84, 1999.

[6] H.K.Lee and D.S. Ha, On the generation of test patterns for combinational circuits, Technical Reports 12-93, Dept. of Elec. Eng. Virginia Polytechnic Institute and State University.

[7] I. Bayraktarouglu O. Sinanoglu and A. Orailoglu. Scan power reduction through test data transition frequency analysis. In Proc. Of International Test Conference, pages 844-850, 2002.

[8] C. Laundrault P. Girard, L. Guiller and S. Pravossoudovitch, A test vector ordering technique for switching activity reduction during test operation. In IEEE Great Lakes Symp. On VLSI, pages 24-27, 1999.

[9] C. Laundrault, Y. Bonhomme, P. Girard and S. Pravossoudovitch,Power driven chaining of flip-flops in scan architectures. In Proc. IEEE International Test Conference, pages 786-803, 2002.

[10] Ozgur Sinanoglu and Alex Orailoglu, A Novel Architecture of Power-Efficient, Rapid Test, International Conference on Computer-Aided Design (ICCAD '02), pages 299-303, 2002.

[11] H. K. Lee and D. S. Ha, An Efficient Forward Fault Fault Simulation Algorithm Based on the Parallel Pattern Single Fault Propagation, Proc. of the 1991 International Test Conference, pp. 946-955, Oct. 1991.

[12] R. Sankaralingam, R. Oruganti and N. Touba, Static Compaction Techniques to Control Scan Vector Power Dissipation, IEEE VLSI Test Symposium, pp 35-42, 2000. 